Tropical Journal of Pharmaceutical Research, December 2002; 1 (2): 83-89

(C) Pharmacotherapy Group Faculty of Pharmacy, University of Benin

Benin City, Nigeria.

All rights reserved.

Available online at http://www.tjpr.freehosting.net

\title{
Evaluation of Dimethylformamide (DMF) as an Organic Modifier in Hydrophobicity Index $\left(\mathbf{R}_{\mathrm{m}}\right)$ Determination
}

\author{
Olakunle S Idowu ${ }^{\dagger}$, Adedigbo A Fasanmade and Ajibola A Olaniyi \\ Department of Pharmaceutical Chemistry, Faculty of Pharmacy, University of Ibadan, Nigeria
}

\begin{abstract}
Purpose: Ideal behaviour of mixtures of organic modifier and water is reflected by a linear relationship between refractive index and fraction of organic modifier in the mixture. This study was carried out to investigate dimethylformamide (DMF) as an organic modifier in hydrophobicity index $\left(R_{m}\right)$ determination.

Method: We quantitatively evaluated the problem of partial miscibility of phases associated with the reversed phase thin layer chromatographic (RPTLC) system, using liquid paraffin as stationary phase and acetone/water mixtures as mobile phase. Ideality of behaviour of acetone /water mixtures was investigated by refractive index measurements. $R_{m}$ values of compounds were determined using mixtures of acetone and water as mobile phase.

Results: DMF/water mixture behaved ideally across the whole concentration range investigated (0-100\%) while acetone/water mixture deviated from ideal behaviour when the concentration of acetone in the mixture was $80 \%$. DMF also gave a better extrapolation of $R_{m}$ value from linear regression of partition data than acetone for bezafibrate used as a test-drug molecule.

Conclusion: DMF is a better organic modifier than acetone in this RPTLC system. These findings could be extended to drug-receptor and drug design studies. The use of dimethylformamide (DMF) in preference to acetone as organic modifier is proposed in this study.
\end{abstract}

Keywords: Drug design, dimethylformamide, hydrophobicity index, organic modifiers

\footnotetext{
${ }^{\dagger}$ To whom correspondence should be addressed: E-mail: olakunleid@yahoo.com Tel: + 2348023094981
} 


\section{Introduction}

Drug design is a multi-disciplinary branch of medicinal chemistry. A quantitative approach to drug design involves correlation of chemical and physical properties with biological activity. Advances in this procedure have demonstrated the effectiveness of using quantitative models of structure-activity relationship (SAR) in describing drug action ${ }^{1}$. The objectives of this exercise are first, to provide an insight into how and why a particular drug possesses the activity for which it is known and second, to make efficient predictions of the chemical structures of drug candidates that will have higher therapeutic efficacy ${ }^{2}$. Progress in the use of quantitative structure activity relationship (QSAR) techniques has shown the importance of the hydrophobic or lipophilic nature of drug molecules ${ }^{3}$. The lipophilicity of a drug is a measure of the ability of a drug molecule to traverse the lipid bilayer of cell membranes and get to its site of action after administration ${ }^{4}$.

Lipophilicity is usually characterized by partition coefficient $(P)$, which is essentially obtained from distribution studies of the drug between an immiscible polar and a non-polar solvent pair. Besides the conventional "shake-flask" method of determining $\mathrm{P}^{5}$, chromatographic methods have also being employed. In the high performance liquid chromatography (HPLC) method $^{6-7}$, the capacity factor ( $\left.\mathrm{K}^{\prime}\right)$ is related to the partition coefficient, P. Biagi et al. ${ }^{8-9}$ have also reported the suitability of reversed phase thin layer chromatography (RPTLC) as a method of determining the lipophilic character of drugs. This was expressed by the term "hydrophobicity index" $\left(R_{m}\right)$. The $R_{m}$ value is also related to $P$. $R_{m}$ is obtained from the TLC parameter, $R_{f}$, by the expression:

$$
R_{m}=\log \left[1 / R_{f}-1\right]
$$

The RPTLC method combines advantages of simplicity, rapidity, low-cost and versatility of application among the various methods.
Reversed phase TLC system uses silica plates impregnated with liquid paraffin, heavy oils, fats or silicone oil and mobile phases containing water. Sometimes, the silica plates have hydrocarbon chains chemically bonded to the hydroxyl groups of silica $^{10}$. The use of $100 \%$ water as the mobile phase is not always successful owing to too high or too low solute polarity. For accuracy and retention of the additivity of the $R_{m}$ values as proposed by the $R_{m}$ theory, $R_{f}$ values between 0.15 and 0.88 are recommended in literature ${ }^{11}$. Adding certain organic modifiers to water alters the polarity of mobile phases. This is done in order to achieve reasonable solute migration that fits into the reliable range. Acetone, methanol and ethanol have been employed in this respect ${ }^{3}$. The use of $5 \%$ liquid paraffin on silica gel and acetone/water mixture as mobile phase is a very popular chromatographic approach employed for $R_{m}$ determination $^{12}$.

Rekker ${ }^{11}$ reviewed the application of RPTLC to partition data generation and identified the fact that the RPTLC system is essentially more complex than the situation normally encountered in the conventional method which uses a simple solvent pair. The complexity is based on the fact that the presence of acetone will partially abolish the immiscibility of the paraffin oil-water system. In consequence, there is a shifting of partitions in favour of the aqueous solvent partner and a decrease in $R_{m}$ values.

In this paper, we report a quantitative evaluation of this "acetone-effect" on the integrity of reversed stationary phase provided by liquid paraffin. The suitability of another organic modifier, a polar aprotic solvent, dimethylformamide (DMF) is proposed for $\mathrm{R}_{\mathrm{m}}$ determination.

\section{Experimental}

\section{Reagents}

Acetone, methanol, liquid paraffin, dimethylformamide and petroleum spirit $\left(40-60^{\circ} \mathrm{C}\right)$ 
(British Drug Houses, UK); iodine crystals (Harris Reagent, UK), p-hydroxybenzoic acid (PHBA), 3,5-dinitrobenzoic acid (DNBA), 3,5dinitrosalicylic acid (DNSA) and silica gel $\mathrm{G}_{6}$ (BDH, UK), ambucetamide, baclofen, bezafibrate andgemfibrozil (Sigma, UK).

\section{Equipment}

Analytical balance (Mettler, AE 160), refractometer, sodium D-lamp (Bellingham Stanley, UK), laboratory centrifuge (Gallenkamp, UK), spinmix (Gallenkamp, UK).

\section{Method}

Thin layer of silica gel $(0.5 \mathrm{~mm})$ was prepared on glass plates as previously described $^{10}$. The plates were coated with liquid paraffin by ascending development as previously described ${ }^{13}$, allowed to air dry and stored in a desiccator before use. Mobile phases used consisted of various mixtures of acetone and dimethylformamide (DMF) with water as well as the pure organic solvents. Solutions $(1 \mathrm{mg} / \mathrm{ml})$ of the compounds; $p$ hydroxybenzoic acid (PHBA), 3,5dinitrobenzoic acid (DNBA), 3,5dinitrosalicylic acid (DNSA), ambucetamide, baclofen, bezafibrate, gemfibrozil, were made in ethanol and applied to the plates with capillary tubes. Intervals of $2 \mathrm{~cm}$ and 1 $\mathrm{cm}$ were allowed between spots on $20 \times 20$ $\mathrm{cm}$ and $10 \times 5 \mathrm{~cm}$ plates respectively. The spotted plates were air-dried and developed in saturated chambers. Developed plates were afterwards visualized in iodine tank.

\section{Miscibility of acetone/water and liquid paraffin}

In order to determine the extent of leaching of liquid paraffin layer on the RPTLC by acetone, mobile phases were prepared to contain $75 \%, 80 \%, 90 \%$ and $95 \%(\mathrm{v} / \mathrm{v})$ of acetone in water and 100\% acetone. Coated plates were developed, without any compounds spotted, in the mobile phases and afterwards kept in iodine tank for $90 \mathrm{~min}$. Extent of leaching of paraffin coating was determined by measuring the leached portion, which appeared as a light brown portion in a mostly dark brown plate surface. The whole procedure was repeated for DMF/water mixture.

Ideality of behaviour of mobile phase solvent mixtures

In order to investigate the ideality of behaviour of the solvent mixtures used as mobile phase, refractive index measurements were carried out. Mixtures of acetone and DMF with water were prepared to contain $5 \%, 10 \%, 20 \%, 40 \%, 50 \%, 60 \%$, $70 \%, 80 \%, 90 \%$ and $100 \%$ of the organic solvents, respectively. Refractive indices (RI) of the solutions were measured and a plot of $\mathrm{RI}$ against fraction of organic modifier was made to determine linearity and hence ideality of behaviour.

\section{Determination of hydrophobicity $\left(R_{m}\right)$ values}

DMF and acetone were used in turn as organic modifier for the determination of $R_{m}$ values. $R_{m}$ values were determined for; $p$ hydroxybenzoic acid, 3,5-dinitrobenzoic acid, 3,5-dinitrosalicylic acid, ambucetamide, baclofen, bezafibrate and gemfibrozil using $0 \%, 25 \%, 50 \%, 75 \%$ and $100 \%$ organic solvent/water mixtures as mobile phase. The mobile phase composition over which the $R_{m}$ theory is applicable was determined by the linear range in the plot of $R_{m}$ versus fraction of organic modifier in the mobile phase.

Mobile phases were prepared to contain $5 \%$, $10 \%, 15 \%$ and $20 \%$ of the organic modifier. The $R_{m}$ values determined for bezafibrate in the various mobile phases were subjected to linear regression analysis. Theoretical $R_{m}$ value at $100 \%$ water was obtained by extrapolation of the regression line. This $R_{m}$ value was compared with an experimentally determined $R_{m}$ value for bezafibrate, using $100 \%$ water as mobile phase.

\section{Results}

The leaching effect of acetone component in acetone/water mixtures is shown in Table 1. 
$100 \%$ DMF and other composition of DMF/water mixture had no leaching effect on the liquid paraffin coating on the RPTLC plates. Acetone/water mixture deviates from linearity around $80 \%$ acetone as monitored by refractive index measurement. DMF/water mixture exhibited biphasic linearity, showing a higher slope between $50 \%$ and $100 \%$ than between $5 \%$ and $50 \%$ of DMF (Figure 1 ). DMF/water mixture gave a similar pattern with acetone/water in the $R_{m}$ data obtained for the various compounds studied (Figures 2 and 3$)$.

Table 1: Leaching effect of acetone on the integrity of RPTLC

\begin{tabular}{ccc}
\hline $\begin{array}{c}\text { Concentration of } \\
\text { acetone (\%) }\end{array}$ & $\begin{array}{c}\text { Distance leached } \\
\text { from surface of } \\
\text { mobile phase }(\mathrm{x})\end{array}$ & $\mathrm{S}_{\mathrm{f}}$ \\
\hline 75 & $0^{*}$ & 0 \\
80 & 0.20 & 0.03 \\
90 & 0.60 & 0.09 \\
95 & 1.70 & 0.24 \\
100 & 3.60 & 0.51 \\
\hline
\end{tabular}

The distance of solvent travel from mobile phase surface $(y)=7.0 \mathrm{~cm}$. $S_{f}=x / y$

*The portion of TLC plates immersed in the mobile phase during development was leached

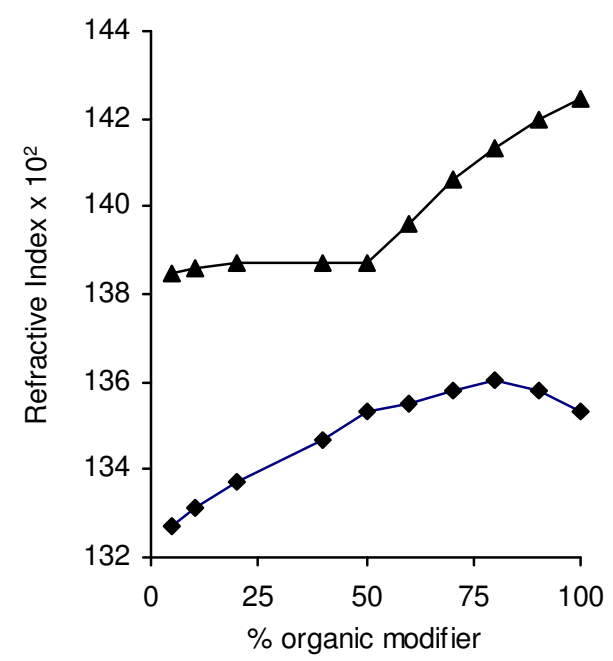

Figure 1: Plot of refractive index (RI) versus percentage of acetone and DMF

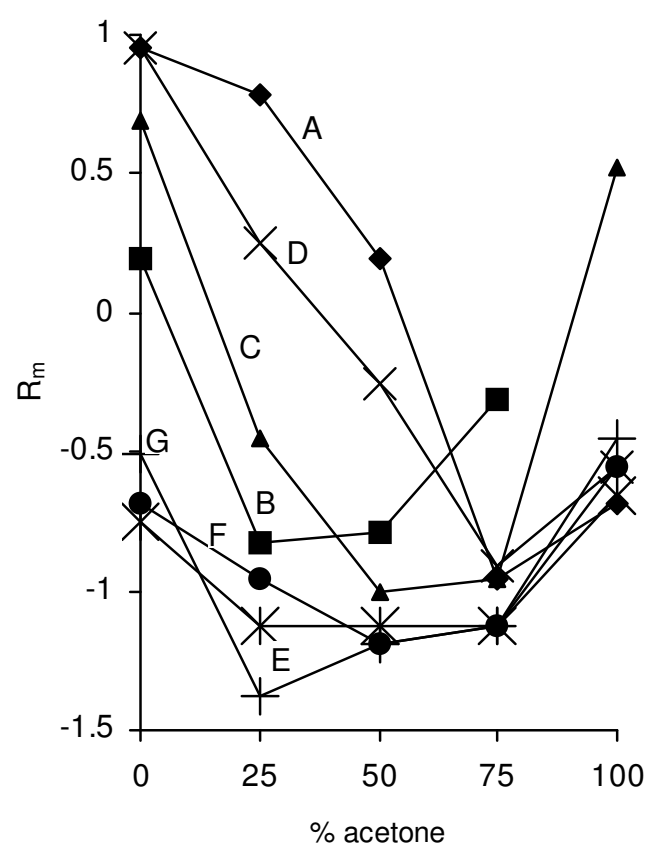

Figure 2: Plot of $R_{m}$ versus concentration of acetone in the mobile phase (A, Ambucetamide; B, Baclofen; C, Bezafibrate; D, Gemfibrozil; E, 3,5-DNBA; F, 3,5-DNSA; G, PHBA)

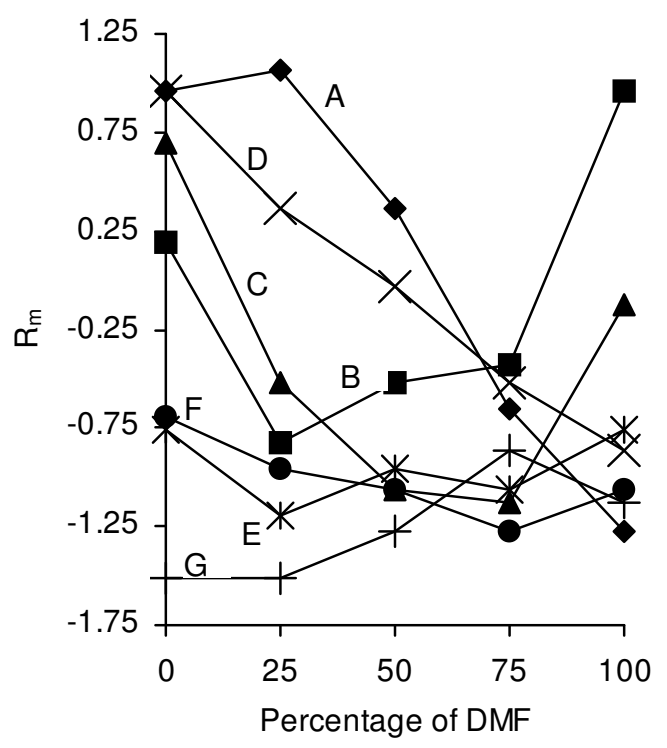

Figure 3: Plot of $R_{m}$ versus concentration of DMF in the mobile phase (A, Ambucetamide; $B$, Baclofen; C, Bezafibrate; D, Gemfibrozil; E, 3,5DNBA; F, 3,5-DNSA; G, PHBA) 
Using bezafibrate as a model compound that gives reasonable solute migration when $100 \%$ water was used as mobile phase, the $R_{m}$ value determined experimentally was 0.689 . The extrapolated $R_{m}$ values obtained for bezafibrate by the two mobile phases were 0.812 and 0.373 for DMF/water and acetone/water respectively (Table 2 ).

Table 2: Regression analysis of $R_{m}$ values against concentration of organic modifier for bezafibrate

\begin{tabular}{|c|c|c|}
\hline \multirow{2}{*}{$\begin{array}{c}\text { Organic } \\
\text { modifier } \\
(\%)\end{array}$} & \multicolumn{2}{|c|}{$\mathrm{R}_{\mathrm{m}}$} \\
\hline & Acetone & DMF \\
\hline 5 & 0.231 & 0.454 \\
\hline 10 & -0.105 & 0.389 \\
\hline 15 & -0.158 & -0.105 \\
\hline 20 & -0.389 & -0.327 \\
\hline $\begin{array}{l}\text { gression ec } \\
\text { Acetone: } \\
\text { of slope }= \\
90 \% \text { confic } \\
0.373 \pm 0.2 \\
\text { DMF: } y= \\
\text { slope }=0 . \\
\text { confidence } \\
0.812 \pm 0.4\end{array}$ & $\begin{array}{l}.0383 x+0 \\
73 ; \text { sd of in } \\
\text { limit for } F \\
67 x+0.81 \\
\text { sd of inte } \\
\text { for } R_{m} \text { (in }\end{array}$ & $\begin{array}{l}=0.9318 ; \text { sd } \\
=0.1002 ; \\
\text { cept })= \\
0.9291 ; \text { sd of } \\
0.1519 ; 90 \% \\
=\end{array}$ \\
\hline
\end{tabular}

\section{Discussion}

The results of the miscibility experiments (Table 1) show that acetone partially abolishes the immiscibility between water and liquid paraffin. In the leaching experiment, the lower portion of the TLC plates where the liquid paraffin had been leached gave a lighter brown shade than the remaining part when kept in iodine tank. On the other hand DMF appears not to affect the immiscibility of water and liquid paraffin. There was no leaching of the paraffin coating on RPTLC plates. This pattern is due to the fact that DMF is much more polar than acetone. It has dielectric constant $(\varepsilon)$ of 36.7 as opposed to 20.7 for acetone. The dipole moment $(\mu)$ is 3.86 for DMF which is higher than 2.88 for acetone ${ }^{14}$. The dipole moment and dielectric constant are both indices of solvent polarity.
In the leaching experiment, the usual practice of pre-saturation of mobile phase with liquid paraffin was not followed, hence the leaching effect observed. It follows therefore that, when acetone/water mixture is pre-saturated with liquid paraffin, the mobile phase is actually made up of three components, liquid paraffin being the third. Its presence in the eluate will inevitably influence the migration of compounds on the chromatoplate. The presence of liquid paraffin in the mobile phase partially negates the essence of extrapolation of the various $R_{m}$ values in order to obtain a theoretical $R_{m}$ value. This is because the stationary phase is now shown to be a component of the mobile phase, once there is pre-saturation with liquid paraffin.

Significant leaching of liquid paraffin begins with $80 \%$ acetone /water mixture (Table 1 ). The use of acetone as organic modifier in $R_{m}$ determinations is therefore unsatisfactory at and beyond $80 \%$ acetone. Biagi et al. ${ }^{8}$ reported that the linear range in $R_{m}$ versus organic solvent fraction plot is valid for extrapolation, only, if the solvent mixtures do not deviate markedly from ideal solutions. In ideal solutions, a linear relationship should exist between the refractive index and concentration of each component. Figure 1 shows that linearity (ideal behaviour) exists between $0 \%$ and $80 \%$ acetone. On the other hand linearity exists throughout the whole range for DMF/water mixture, although the graph is biphasic in nature. This is because the rate of change in refractive index with change in fraction of DMF is higher beyond $50 \%$ DMF than below it.

Determination of $R_{m}$ value was carried out in each of the two solvent systems. The compounds used include acidic and basic drugs of varying chemical structures (Figure 4). Validity of $R_{m}$ values are only assumed if the $R_{f}$ of a compound in a particular solvent system falls within the range 0.15 to 0.88 . This corresponds to $R_{m}$ values of -0.87 to +0.75 . The miscibility of liquid paraffin with acetone/water mixture with increasing amount of acetone obviously affects linearity 
Idowu et al

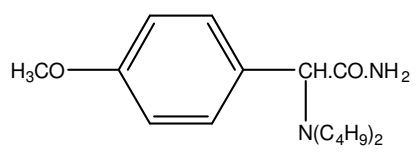

Ambucetamide (antispasmodic agent)

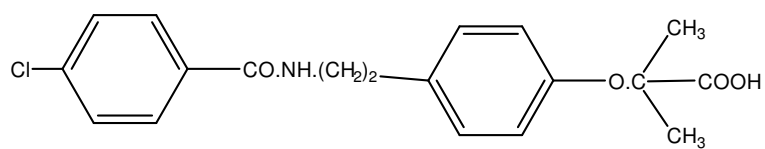

Bezafibrate (antihyperlipoproteinemic agent)

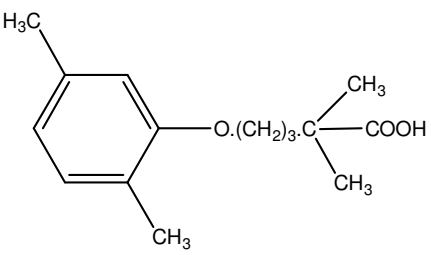

Gemfibrozil (antihyperlipoproteinemic agent)

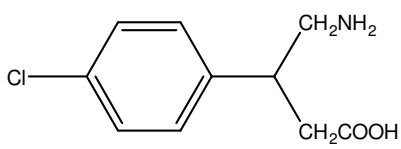

Baclofen (muscle relaxant)
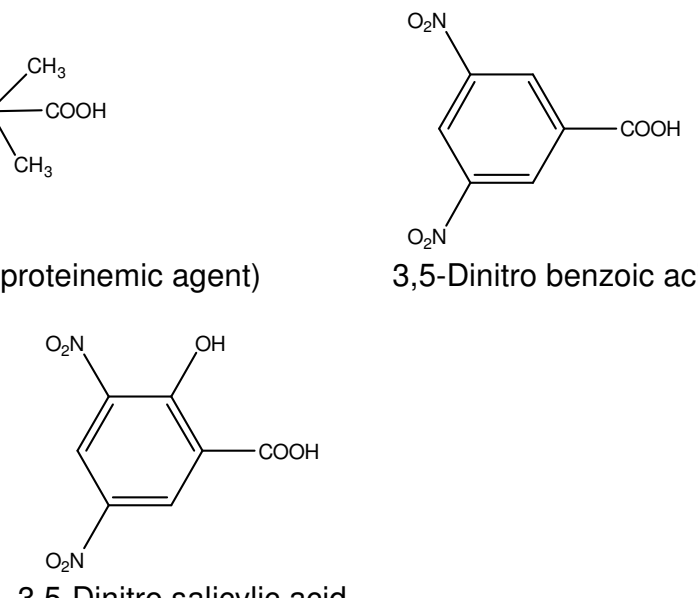

3,5-Dinitro benzoic acid

\section{3,5-Dinitro salicylic acid}

Figure 4: Chemical structures of compounds studied

(Figure 2). With the use of DMF as organic modifier, linear range was obtained over concentration range similar to that of acetone.

Bezafibrate is a model compound that gave reasonable migration with $100 \%$ water as mobile phase. Experimentally determined $R_{m}$ value of 0.689 was obtained. Extrapolated $R_{m}$ value from regression analysis of $R_{m}$ data over a linear range gave $90 \%$ confidence limit of $0.812 \pm 0.44$ and $0.373 \pm 0.29$ for $\mathrm{DMF} /$ water and acetone/water mixture, respectively. The theoretical $R_{m}$ extrapolated from the two sets of data differs from the experimental value. The value obtained from acetone/water differs by $45.86 \%$ relative deviation while the $R_{m}$ value obtained from DMF/water differs by $17.85 \%$ relative deviation. The DMF/water gave a better prediction of the theoretical $R_{m}$ value.
The $R_{m}$ value is linearly related to octanol/ water partition coefficient $(\log P)^{15}$ by the relationship:

$$
\log P=R_{m}+\text { constant }
$$

Values of $\log P$ are typically employed to compute $\pi$ values, which are used to estimate the hydrophobic nature of substituents on an aromatic ring. This is determined as the difference in the $\log \mathrm{P}$ of a substituted analog and the parent compound in the series of compounds under investigation. The computed $\pi$ value is afterwards used as a quantitative chemical descriptor employed in QSAR multiple regression equations for prediction of biological activity ${ }^{16}$.

DMF is a high boiling solvent (boiling point = $153^{\circ} \mathrm{C}$ ) as opposed to relatively volatile 
Idowu et al

acetone (boiling point $=56^{\circ} \mathrm{C}$ ), hence there is good reproducibility within runs since composition of mobile phase does not readily alter on standing. This could also contribute to the accuracy of predicted $R_{m}$ values as well as the precision.

\section{Conclusion}

Acetone, which is widely used as organic modifier for $\mathrm{R}_{\mathrm{m}}$ determination, has certain limitations. At higher concentrations (above $80 \%$ ) it interferes with the predominant partition process in the RPTLC system by leaching liquid paraffin from the stationary phase. If the mobile phase is pre-saturated with liquid paraffin, a more complex three component mobile phase is employed in effect. This reduces the linear range of $R_{m}$ versus acetone fraction plot. DMF has better solubility properties; it is more polar and behaves more ideally. It gave a wider linear range in $R_{m}$ versus organic modifier fraction plot. It has greater flexibility of use and more efficient prediction of theoretical $R_{m}$ for a test drug. The use of DMF could therefore find wider application in $\mathrm{R}_{\mathrm{m}}$ determination and drug design studies.

\section{Acknowledgement}

Part of this work was carried out with the Senate Research Grant SRG/COM/1996/ 43A of the University of Ibadan, awarded to SOI. We thank Mr. AO Adegoke for his technical assistance.

\section{References}

1. Olaniyi AA. Quantitative structure activity relationship. In: Essential Medicinal Chemistry, $2^{\text {nd }}$ ed. Ibadan, Nigeria: Shaneson Cl Limited, 2000 pp 128-142.

2. Tomlinson E. Chromatographic hydrophobic paramters in correlation analysis of structure activity relationships. J Chromatogr 1975; 113: $1-45$.
3. Fasanmade AA, Olaniyi AA, Yisak WAB Mathematical models of antisickling activities of benzoic acid derivatives on red blood cells of sicklers. Afr J Med Medical Sci 1994; 23: 36977.

4. Kalizan R. Chromatography in studies of quantitative structure activity relationships. J Chromatogr 1981; 220: 71-83.

5. Leo A, Hansch C, Elkins D. Parition coefficient and their uses. Chem Rev 1971; 71: 525-55.

6. Twitchet P, Moffat AC. High pressure liquid chromatography of drugs: An evaluation of octadecylsilane stationary phase $\mathrm{J}$ Chromatogr 1975; 111: 149-57.

7. Carlson RM, Carlson RE, Kopperman $\mathrm{HL}$. Determination of partition coefficients by liquid chromatography. J Chromatogr 1975; 107: 21923

8. Biagi GL, Barbaro AM, Gamba AF, Guerra MC Partition data of penicillins determined by means of reversed phase thin layer chromatography. J Chromatogr 1969; 41: 371-9.

9. Biagi GL, Barbaro AM, Gamba MF, Guerra MC Partition data of cephalosporins determined by means of reversed phase thin layer chromatography. J Chromatogr 1969; 44: 195-8.

10. Moffat AC. Thin layer Chromatography. In: Moffat AC. Clarke's isolation and determination of drugs, $2^{\text {nd }}$ ed. London: The Pharmaceutical Press, 1986 pp 160-1.

11. Rekker RF. The position of reversed phase thin layer chromatography amongst solvent partitioning techniques. J Chromatogr 1984 300: 109-25.

12. Biagi GL, Guerra MC, Barbaro AM. Relationship between lipophilic character and haemolytic activity of testosterone and testosterone esters. J Med Chem 1970; 13: 1944

13. Stahl E. Thin layer Chromatography, $2^{\text {nd }}$ ed. New York: Springer-Verlag, 1969 p 227.

14. Gordon AJ, Ford RA. Chemists Companion. John Wiley \& Sons Inc., 1972 pp 6-7.

15. James KC. Quantitative Structure Activity Relationships and drug design. In: Smith $\mathrm{HJ}$ Williams $\mathrm{H}$. (ed.). Introduction to the Principles of Drug Design, $1^{\text {st }}$ ed. London: John Wright \& Sons Ltd., 1983 pp 226-7.

16. Maliski EG, Bradshaw J. QSAR and the role of computers in Drug Design. In: Ganellin CR Roberts SM (ed). Medicinal Chemistry: The role of Organic Chemistry in Drug Research, $2^{\text {nd }}$ ed. London: Academic Press, 1993 pp 94-102. 


\section{Useful Online Full-Text and Abstract Reference Sources}

Pharmacotherapy Group website

http://www.prg.faithweb.com

University of Benin Library

http://www.uniben.edu/ 